\title{
Technology and Care for Patients with Chronic Conditions: The Chronic Care Model as a Framework for the Integration of ICT
}

\author{
Nick Guldemond $^{1}$ and Magda David Hercheui ${ }^{2}$ \\ ${ }^{1}$ Delft University of Technology, Faculty of Electrical Engineering, \\ Mathematics \& Computer Science, Dept. Interactive Intelligence, Netherlands \\ N.A.Guldemondatudelft.nl \\ ${ }^{2}$ Westminster Business School, London, United Kingdom \\ m.hercheui@westminster.ac.uk
}

\begin{abstract}
Worldwide, healthcare systems are considered unsustainable due to an increase in demand for care and an associated rise in healthcare costs. Ageing of societies and the growth of populations with chronic conditions are making a paradigm shift in western healthcare systems necessary. The Chronic Care Model (CCM) provides a framework for healthcare change, including a prominent role for the community and patients' self-management. Information and communication technology (ICT) is indispensable to accomplish the model's objectives. The role of ICT in the provision of care is discussed as an opportunity to facilitate the application of the CCM and improve healthcare in general.
\end{abstract}

Keywords: healthcare, ICT, innovation, integrated care, technology.

\section{Introduction}

The ageing of society is a key societal challenge for many countries worldwide. The prevalence of chronic diseases is rising globally due to the ageing of societies in combination with the effects of a sedentary life style. This trend results in a rise of healthcare costs an enormous pressure on the resources in society [31]. Implementation of healthcare services where medical and social aspects are integrated is imperative as well as the use of technology to facilitate self-management and cost-effective service delivery. When they grow older, people are more prone to multiple and chronic ailments. Chronic diseases such as cardiovascular disease, diabetes, arthritis and dementia are among the most common, costly, and preventable of all health problems in the world and are leading causes of death and disability. Currently the working population is shrinking. Projections show a growing imbalance between the number of elderly citizens in need of care and the actual supply of formal care services ${ }^{1}$. This is indicated by the age dependency ratio $^{2}$, which shows an increasing pressure on the

\footnotetext{
${ }^{1}$ http: / / europa.eu.int/comm/economy_finance/ epc/epc_publications_en.htm

${ }^{2}$ http://esa.un.org/wpp/Sorting-Tables/tab-sorting_ageing.htm
} 
productive population of many countries ${ }^{3}$. In 2007, the age dependency ratio of the European Union (EU) was on average one retired citizen to four working people. Without anticipatory action, the average in 2050 will be an estimated 1:2. In the Netherlands, there will be an average ratio of 1:3 and in Italy and Spain an average ratio of 1:1.5, while in China this age dependency ratio will be less than 1 . By 2025 the EU will lose an average of one million workers a year, while an increasing number of people has to be employed in healthcare. By then, about $25 \%$ of the working population should be employed in healthcare in the Netherlands, while optimistic scenarios for Greece, Italy and Spain will approach 50\% [43]. The consequence of this growth in both chronic diseases and ageing is an increasing demand on healthcare while financial and human resources are decreasing. Current western healthcare systems are considered to be unsustainable, since the institutional care provision maintains a focus on curing diseases rather than preventing them. We can no longer expect that formal carers and professional institutions will provide the services for the support of an increasing number of people with a chronic condition.

In a complex health system, the management of chronic illness requires the coordination of care. Historically, there has been little coordination across the multiple settings, providers and treatments of chronic illness care. Integrated care may be seen as a response to the fragmented delivery of health and social services. The proposed paradigm shift in healthcare systems comprises a transition: 1) from mainly a monodisciplinary to a multi-disciplinary form of care provision, 2) from a curative approach to preventive medicine and public health, 3 ) from institutional care to community care, and 4) from professional care to informal care.

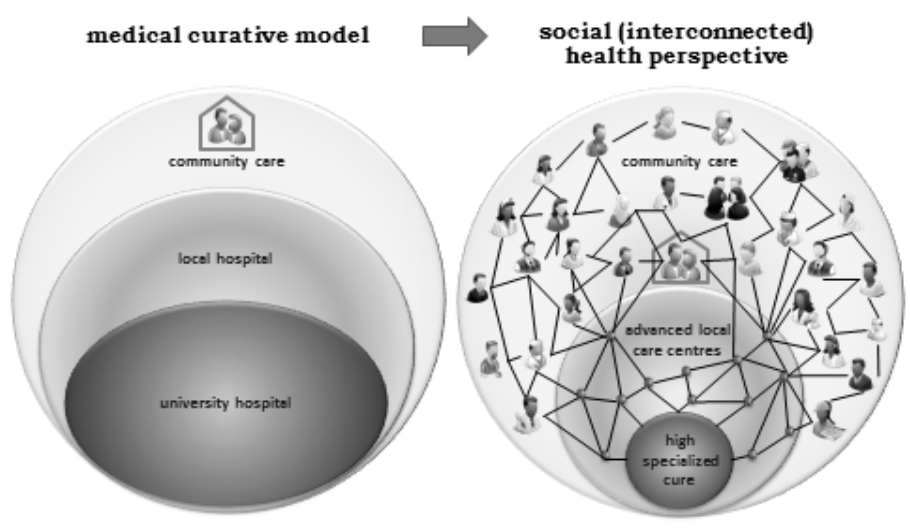

Fig. 1. Paradigm shift in healthcare systems

The concept of healthcare practice and healthcare change is based on frameworks or models. Healthcare models are a set of policies and organizational arrangements for meeting the three basic goals of a healthcare system: keeping people healthy, treating people who are sick, and protecting citizens against the financial burden as a

3 http://www.euphix.org/object_document/o5117n27112.html 
result of the amount of care that is consumed. Hence, these models provide the framework that should guarantee accessibility, quality and affordability of healthcare and ensure that patients get the optimal care, at the right time, by the right people and in the right place.

\section{The Chronic Care Model and Integrated Care}

In the search for effective strategies to prevent and manage chronic diseases, different models and programs have been proposed such as the Disease Management Program [22], Shared Care Program [23], [39], Chronic Care Model [2], [46], and Guided Care Model [6], [33]. In spite of their differences, all these models or programs aimed at a less fragmented, more integrated provision of patient-centered care.

The Chronic Care Model (CCM) by Wagner and colleagues [46] is currently central to European and United States' healthcare policy, and includes important elements of self-management, social support organizations, informal carers and the community. The CCM was intended to be a framework for innovation and improvement of care for chronic conditions. The CCM was later refined by Barr and colleagues [2], so that it became i.e. "the expanded CCM" that accentuated prevention and health promotion. The CCM assumes that good health outcomes require productive interactions between a multi-disciplinary team of professionals and an active patient and his or her proxies, such as families and informal caregivers. This interaction is the result of a form of coordinated care that is composed of six distinctive elements along with its relevant aspects, as laid out in the CCM (figure 2). There are two contextual elements: 1) the community and 2) the healthcare system. Within the healthcare system there are four focus areas for innovation: 3) the delivery system, 4) information systems, 5) decision support and 6) self-management. In the community element, Barr and colleagues emphasized the necessary community resources and policies that facilitate concrete actions, as well as the importance of public health and a supportive environment [2]. While technology is mentioned in most healthcare models, further elaboration on the required functionalities remain absent, as well as the implications of future information and communication technology (ICT) developments.

In this paper, the role of ICT and future developments are discussed from the perspective of CCM and its related components (figure 2), as follows. Health System: program planning (including measureable goals for better care of chronic illness) to meet the health needs of target populations. Community: mobilizing people to engage in informal care and developing partnerships with community organizations that meet the needs of patients with chronic conditions. Delivery system: the process of reorientation of health services with a focus on multidisciplinary teamwork drawing on individuals and skills from the health and social care sectors. Information system: smart information systems based on patient populations to provide all the relevant client data (medical, health and social). Decision support: integration of evidencebased medicine, protocols and guidelines into a holistic approach. Self-management: interventions, training, and skills by which patients with a chronic condition can 
effectively take care of themselves. Note: health system and community are dealt with in two separate sections below, where the section on "health system" explores the notions of information systems, decision support, delivery system and selfmanagement. The text on the health system is treated purely as background and is not explored in detail.

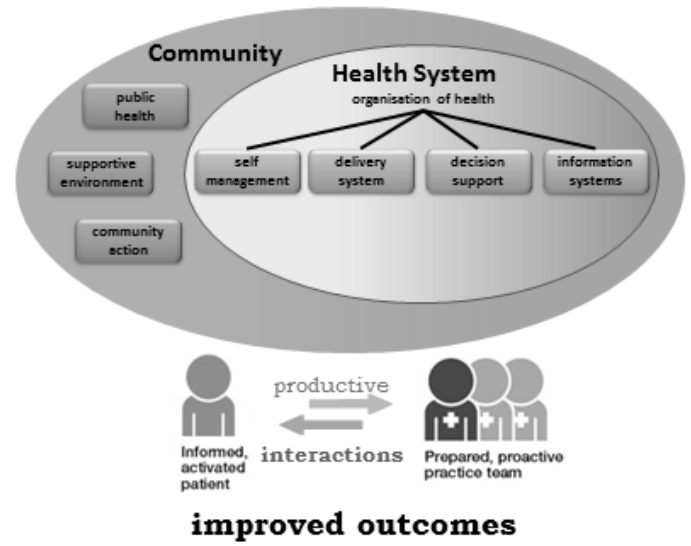

Fig. 2. Expanded Chronic Care Model: adopted from Barr et al. [2]

\section{$3 \quad$ Health System}

The health system is the operational body that translates healthcare policy into practice through programs that subsume and support the management, define the performance indicators in relation to the standard of healthcare quality, and formulate the related business plan. The health system comprises information systems, decision support, the delivery system and self-management.

\subsection{Information Systems}

Health information systems provide a means of making the information about an entire patient population (a registry) available to the patient and provider [30]. Over the last decades, the digital revolution has made an enormous change in the availability of clinical information. This accounts for traditional information sources such as patient records, daily practice data (e.g. laboratory results and radiographs) as well as protocols, guidelines and science-related information. Clinicians also have increasingly more access to animated information on patients' physiology, anatomy and pathology.

Besides these developments in bio-medical based practice, there is a current trend of collecting personal health or life style data. This is made possible through common devices such as weight scales, blood pressure and glucose monitors that communicate through the Internet and through a variety of innovative diagnostic tools for home care. Portable devices and smartphones also enable monitoring of activity, sleep and 
food consumption. Millions of individuals are storing streams of personal data through their websites, cell phones, laptops and other digital devices, which might be used to predict and anticipate conditions, behaviors and events relevant to their health.

For adequate healthcare management, an integration of bio-medical, health and social care data is needed, including community data [2]. In chronic care management, relevant users of information systems are also patients, informal carers, advocacy groups, municipalities and everyone who is indispensable in the provision of care. This requires data integration of various sources, data analysis and data stewardship with which intelligent agent software can help [28], [38], [40]. Software agents that incorporate learning, context-sensitivity, personalization, collaboration and proactivity will enable a support system that provides tailored medical information for practice teams [4]. For patients and informal carers, agent-based technology could offer personalized information with alerts, recalls and instructions for health promotion. Agent technology is also useful for population data-mining to identify subgroups that need proactive care, and to see whether preventive policy measures have an effect on primary medical, life style and social indicators [44]. Data technologies are becoming so important in providing solutions for societal challenges that government and citizens have to address urgently questions about protection from the misuse or abuse of large databases in a wide number of areas that also include health and healthcare. Besides the technological solutions for data protection and privacy, new regulations and legislation are needed. Data privacy should be seen in a wider context than just secure data transfer and information sharing among healthcare professionals.

\subsection{Decision Support}

Decision support provides healthcare professionals with instructions, guidelines and protocols to ensure that best evidence-based care is delivered. Decision support is strongly dependent on the expertise of the clinician, clinical information systems and evidence from medical research. As described in section 3.1, most important information needed for decision support is digitally available, but it is fragmented and only partially used. The overwhelming amount of clinical information generally available has made it much more difficult to extract useful information, and keep up with the progress in particular medical disciplines [35]. Considering the limited time for consultation by a clinician, it is unrealistic that all information regarding a specific patient can be part of the clinical reasoning process. To translate data into practice, clinicians typically have to: 1) formulate questions that are answerable from the information sources, 2) search for and identify evidence to answer the clinical questions and 3) appraise the evidence identified for quality, reliability, accuracy and relevance [36]. A clinician or a practice team would be greatly supported by an automated clinical reasoning support tool in which available evidence from the literature is selected, deliberated on, summarized, and attuned with guidelines and treatment protocols [16]. A patient's profile, derived from both medical and social data, could form a basis for personalization through which the available evidence could be tailored into relevant instructions for each person involved in the care process. Automated decision support is an important facilitating tool for integrated care. 


\subsection{Delivery System}

The delivery system of healthcare addresses the overall workflow coordination. Highquality healthcare requires certain elements for adequate performance. The most vital elements in the delivery system dedicated to integrated care are well-trained and motivated people, accurate information, and technologies that enable providers to use resources effectively. This involves cross-sectorial primary, secondary and social care work flow planning, communication and quality control.

Planning in a delivery system deals with 1) who should be on a care team, 2) what kind of interaction each member of the team should have with the patient, 3) how team members should interact with each other, and 4) how patients communicate with caregivers. Efficient planning and harmonizing tasks of formal and informal carers is crucial for effective care [8]. Software tools for care management can help with the scheduling of appointments and services such as case management.

Communication in the delivery system is indispensable for the collaboration between stakeholders. Chronic care needs collaboration between primary and informal care (in the community) as well as secondary care (in an institution). The organizational structure of distinctive primary and secondary care sectors hampers the provision of integrated care [9], [14]. Due to the existence of so many unique specialisms and disciplines, horizontal communication among different medical specialists in secondary care and vertical communication between primary and secondary care is problematic [19].

Computerized health information systems could overcome most cross-sectorial communication issues. However, the implementation of shared information systems is far behind that seen in other sectors and industries. Despite the overwhelming evidence of the cost-effectiveness of multi-disciplinary use of electronic medical records, email, text-messages, social media and video communication services such as Skype [37], [51]. Many technology-related reasons have been mentioned as obstacles to the use of health information systems, including security, privacy, incompatibility between systems, maintenance, upgrades, ease of use and lack of integration with other applications [17]. Web-based applications and solutions provided by cloud computing as an alternative to local hosted software and storage concepts could overcome many of these issues.

Quality control and transparency of data about clinical effectiveness and costeffectiveness of procedures, diagnostic and therapeutic devices and drugs is an increasing societal need. Healthcare consumers might be involved in providing feedback on performance indicators. Patients and their proxies could rate their carers, consumed services and hospitals visited [21]. Accordingly, competitiveness among healthcare providers can be stimulated. With shared information systems and social media it is possible to link healthcare services to patient satisfaction, quality indicators, cost and, ultimately, regional or national benchmarks. 


\subsection{Self-management}

Patient engagement is increasingly important as a solution to lowering costs and improving population health. Self-management aims to enable people to remain independent and active and to support them to live in their own homes as long as possible. Self-management support involves empowering patients and their caregivers through education, training, skills acquisition, and help with integrating problem-solving and goal-setting into everyday care [1], [2], [5], [32]. Many aspects of self-management could be supported by solutions enabled by technologies. There is evidence of improvement of patients' self-management through web-based applications and social media [45]. Most research is focused on technologies for self-monitoring and selfeducation, where changes in patient adherence and levels of knowledge showed significant improvements [10], [24]. Hence, opportunities for further improvement lie in supporting patients to manage their health by finding information about their condition on the web and participating in social networks through which they can exchange information and provide support to each other [3]. This could be supported by intelligent systems that provide personalized assistance by remembering past interactions, and inferring the user's preferences through his or her actions [20].

\section{Community}

Community resources and policies are essential to facilitate the implementation of technology supported integrated care [27]. To implement integrated care according to CCM, new partnerships in the community with citizens, medical and social professionals have to be established. Communication and sharing of information, and alignment of objectives, resources and actions are also essential. Prevention of illness and readmission requires community actions, while informal carers play an important role in maintaining the health and wellbeing of people with chronic conditions. Health is predominantly influenced by the community factors, including the quality of housing, safety, social interaction, and transport. Supportive environments could help dependent and vulnerable people keep actively involved, people who would be otherwise be at risk of losing their independence. Outreach to outlying and underserved communities is being supported through a number of technological approaches [42].

\subsection{Public Health}

The objective of public health is to improve health and quality of life through the prevention and treatment of disease. Public health comprises a multidisciplinary approach, where health promotion is dedicated to enable people to manage their health by education and social marketing focused on changing behavioral risk factors such as a sedentary lifestyle, unhealthy food consumption, mental stress and smoking [7]. Social marketing applies commercial marketing strategies to promote public health in mass communication. The combination of market strategies and social media could influence the attitudes, beliefs and behaviors of communities and individuals [41]. Social media stimulate active participation of the patient, which is an element of 
successful behavioral change [47]. Healthcare providers can contribute to the effectiveness of patient involvement during their direct and indirect contacts with patients [11].

\subsection{Supportive Environment}

Supportive environments provide support for daily life activities and facilitates patients to stay active and independent, including solutions that support people in the work environment, and incorporate home automation through sensor technology and service robots [13], [34]. Smartphones and tablets can enable empowerment, wellness and the provision of social care services to the homes of dependent elderly persons and patients [26], [29]. Interactive multimedia allows a two-way communication for family, friends and caregivers. Agent-based software could enable more sophisticated communication and problem-solving between professional and non-professional carers. Integration of social networking and serious gaming elements into communication services could engage people in sharing community care. Mobilizing local communities for the support of care-dependent people is a great societal need and will have a major positive impact on social cohesion and the economy [15], [18].

\subsection{Community Action}

Informal caregivers are individuals who provide on-going assistance, without pay, for family members and friends in need of support. Informal care is a resource that is under both demographic and economic pressure [25]. Projections show that less informal care will be available within households as a result of trends in family size and scarcity on the labor market (in combination with an increase in the participation of women in the labor market ${ }^{4}$ ). The only solution to these limited resources for the support of vulnerable and dependent people is to organize informal support systems more efficiently. We need mobilization within our communities, thus it is imperative that we "engage all": technologies such as social media could help to achieve this [12].

\section{Conclusion}

The ageing of western society is a great challenge that is having a major impact on healthcare costs and community resources. Overcoming the problems of ageing and chronic conditions can only be achieved by transforming the current healthcare system with an integrated approach where multidisciplinary care teams, self-management and community involvement all play a central role. Technology is essential to this transition. The CCM provides essential health system and community elements into which technology could be integrated purposefully.

\footnotetext{
${ }^{4}$ http: / / ec.europa.eu/economy_finance/publications / archive/special_reports_en.htm
} 
Medical and social information has to be merged into a shared and personalized record, for patients as well as for both professional and informal carers. Decision support could be improved by an integration of evidence-based medicine, clinical expertise and patient values (based on social, medical and cultural profiles) into an automated clinical reasoning system. A cross-sectorial resource management and communication system would overcome the problems of the current siloed structure of social, primary and secondary care sectors. Patients' self-management would benefit from cloud-based computing and mobile technologies. Smart web-based management tools with automated personalized support would be a solution desired by many patients and carers: the most recent data and support tools could be instantly available to provide the most optimal care. Current developments in ICT are the ultimate facilitators for integrated care and the CCM.

\section{References}

1. Barlow, J., Wright, C., Sheasby, J., Turner, A., Hainsworth, J.: Self-management approaches for people with chronic conditions: a review. Patient Education and Counseling 48(2), 177-187 (2002)

2. Barr, V.J., Robinson, S., Marin-Link, B., Underhill, L., Dotts, A., Ravensdale, D., Salivaras, S.: The expanded Chronic Care Model: an integration of concepts and strategies from population health promotion and the Chronic Care Model. Hospital Quarterly 7(1), 73-82 (2003)

3. Bickmore, T., Schulman, D., Yin, L.: Maintaining Engagement in Long-term Interventions with Relational Agents. Engineering Applications of Artificial Intelligence 24(6), 648-666 (2010)

4. Bickmore, T.W., Mauer, D., Brown, T.: Context Awareness in a Handheld Exercise Agent. Pervasive and Mobile Computing 5(3), 226-235 (2009)

5. Bickmore, T.W., Pfeifer, L.M., Byron, D., Forsythe, S., Henault, L.E., Jack, B.W., Silliman, R., Paasche-Orlow, M.K.: Usability of conversational agents by patients with inadequate health literacy: evidence from two clinical trials. Journal of Health Communication 15(suppl. 2), 197-210 (2010)

6. Boult, C., Karm, L., Groves, C.: Improving chronic care: the "guided care" model. The Permanente Journal 12(1), 50-54 (2008)

7. Bunton, R., Macdonald, G.: Health promotion: disciplines, diversity, and developments. Routledge, London \& New York (2002)

8. Butt, G., Markle-Reid, M., Browne, G.: Interprofessional partnerships in chronic illness care: a conceptual model for measuring partnership effectiveness. International Journal of Integrated Care 8, e08 (2008)

9. Castillo, V.H., Martinez-Garcia, A.I., Pulido, J.R.: A knowledge-based taxonomy of critical factors for adopting electronic health record systems by physicians: a systematic literature review. BMC Medical Informatics and Decision Making 10, 60 (2010)

10. Dorr, D.A., Wilcox, A., Burns, L., Brunker, C.P., Narus, S.P., Clayton, P.D.: Implementing a multidisease chronic care model in primary care using people and technology. Disease Management 9(1), 1-15 (2006)

11. Evans, W.D.: How social marketing works in health care. British Medical Journal 332(7551), 1207-1210 (2006) 
12. Fyrand, L.: Reciprocity: A Predictor of Mental Health and Continuity in Elderly People's Relationships? A Review. Current Gerontology and Geriatrics Research (2010)

13. Graf, B., Jacobs, T.: Robots in the nursing home. It gives the impression of something pleasantly service-like. Zeitschrift für die Gesundheits- und Krankenpflege 64(11), 646649 (2011)

14. Granlien, M.F., Simonsen, J.: Challenges for IT-supported shared care: a qualitative analyses of two shared care initiatives for diabetes treatment in Denmark "I'll never use it" (GP5). International Journal of Integrated Care 7, e19 (2007)

15. Harley, D., Kurniawan, S.H., Fitzpatrick, G., Vetere, F.: Age matters: bridging the generation gap through technology-mediated interaction. Extended Abstracts on Human Factors in Computing Systems (2009)

16. Hatzakis, M.J., Allen Jr., C., Haselkorn, M., Anderson, S.M., Nichol, P., Lai, C., Haselkorn, J.K.: Use of medical informatics for management of multiple sclerosis using a chronic-care model. Journal of Rehabilitation Research and Development 43(1), 1-16 (2006)

17. Hayrinen, K., Saranto, K., Nykanen, P.: Definition, structure, content, use and impacts of electronic health records: a review of the research literature. International Journal of Medical Informatics 77(5), 291-304 (2008)

18. Hennessey, B., Suter, P., Harrison, G.: The home-based chronic care model: a platform for partnership for the provision of a patient-centered medical home. Caring 29(2), 18-24 (2010)

19. Hicks, J.M.: Leader communication styles and organizational health. Health Care Management (Frederick) 30(1), 86-91 (2011)

20. Hsu, W., Taira, R.K., El-Saden, S., Kangarloo, H., Bui, A.A.: Context-based electronic health record: toward patient specific healthcare. IEEE Transactions on Information Technology in Biomedicine 16(2), 228-234 (2012)

21. Hvenegaard, A., Arendt, J.N., Street, A., Gyrd-Hansen, D.: Exploring the relationship between costs and quality: does the joint evaluation of costs and quality alter the ranking of Danish hospital departments? European Journal of Health Economics 12(6), 541-551 (2011)

22. Jonker, A.A., Comijs, H.C., Knipscheer, K.C., Deeg, D.J.: Promotion of self-management in vulnerable older people: a narrative literature review of outcomes of the Chronic Disease Self-Management Program (CDSMP). European Journal of Ageing 6(4), 303-314 (2009)

23. Kahan, M., Wilson, L., Midmer, D., Ordean, A., Lim, H.: Short-term outcomes in patients attending a primary care-based addiction shared care program. Canadian Family Physician 55(11), 1108-1109, e1105 (2009)

24. Kaufman, N.: Internet and information technology use in treatment of diabetes. International Journal of Clinical Practice (166), 41-46 (2010)

25. Keefe, J., Legare, J., Carriere, Y.: Developing New Strategies to Support Future Caregivers of the Aged in Canada: Projections of Need and their Policy Implications. Social and Economic Dimensions of an Aging Population (SEDAP). Hamilton, Ontario. RP no. 140 (2005)

26. Lenert, L.: Transforming healthcare through patient empowerment. Studies in Health Technology and Informatics 153, 159-175 (2010)

27. Leykum, L.K., Palmer, R., Lanham, H., Jordan, M., McDaniel, R.R., Noel, P.H., Parchman, M.: Reciprocal learning and chronic care model implementation in primary care: results from a new scale of learning in primary care. BMC Health Services Research 11, $44(2011)$ 
28. Lieberman, H., Mason, C.: Intelligent agent software for medicine. Studies in Health Technology and Informatics 80, 99-109 (2002)

29. Lynch, C.P., Egede, L.E.: Optimizing diabetes self-care in low literacy and minority populations-problem-solving, empowerment, peer support and technology-based approaches. Journal of General Internal Medicine 26(9), 953-955 (2011)

30. McEvoy, P., Laxade, S.: Patient registries: a central component of the chronic care model. British Journal of Community Nursing 13(3), 127-128, 130-123 (2008)

31. McPherson, B., Wister, A.V.: Aging as a social process: Canadian perspectives. Oxford University Press, Oxford (2008)

32. Miller, W., Rollnick, S.: Motivational interviewing: preparing people for change. Guilford Press, New York (2002)

33. Mitchell, G.J., Bournes, D.A., Hollett, J.: Human becoming-guided patient-centered care: A new model transforms nursing practice. Nursing Science Quarterly 19(3), 218-224 (2006)

34. Oborn, E., Barrett, M., Darzi, A.: Robots and service innovation in health care. Journal of Health Services Research \& Policy 16(1), 46-50 (2011)

35. Olkin, I.: Meta-analysis: reconciling the results of independent studies. Statistics in Medicine 14(5-7), 457-472 (1995)

36. Oxman, A.D., Sackett, D.L., Guyatt, G.H.: Users' guides to the medical literature. I. How to get started. The Evidence-Based Medicine Working Group. Journal of the American Medical Association 270(17), 2093-2095 (1993)

37. Piette, J.D., Mendoza-Avelares, M.O., Ganser, M., Mohamed, M., Marinec, N., Krishnan, S.: A preliminary study of a cloud-computing model for chronic illness self-care support in an underdeveloped country. American Journal of Preventive Medicine 40(6), 629-632 (2011)

38. Rialle, V., Lamy, J.B., Noury, N., Bajolle, L.: Telemonitoring of patients at home: a software agent approach. Computer Methods and Programs in Biomedicine 72(3), 257-268 (2003)

39. Sanchez, I.: Implementation of a diabetes self-management education program in primary care for adults using shared medical appointments. Diabetes Educator 37(3), 381-391 (2011)

40. Sayyad Shirabad, J., Wilk, S., Michalowski, W., Farion, K.: Implementing an integrative multi-agent clinical decision support system with open source software. Journal of Medical Systems 36(1), 123-137 (2012)

41. Segbers, R.: Go where the customers are. Marketing (and managing) your patient experience with social media. Marketing Health Services 30(1), 22-25 (2010)

42. Siminerio, L.M.: The role of technology and the chronic care model. Journal of Diabetes Science and Technology 4(2), 470-475 (2010)

43. SPB: Actualisatie en aanpassing ramingsmodel verpleging en verzorging 2009-2030. Den Haag, Sociaal en Cultureel Planbureau (2012)

44. Szalma, S., Koka, V., Khasanova, T., Perakslis, E.: Effective knowledge management in translational medicine. Journal of Translational Medicine 8(1), 68 (2010)

45. Thielst, C.B.: Social media: ubiquitous community and patient engagement. Frontiers of Health Services Management 28(2), 3-14 (2011)

46. Wagner, E.H., Austin, B.T., Von Korff, M.: Improving outcomes in chronic illness. Managed Care Quarterly 4(2), 12-25 (1996)

47. Watson, A., Bickmore, T., Cange, A., Kulshreshtha, A., Kvedar, J.: An internet-based virtual coach to promote physical activity adherence in overweight adults: randomized controlled trial. Journal of Medical Internet Research 14(1), e1 (2012) 